\title{
CHILDREN'S CATEGORY STRUCTURE AND FALSE MEMORIES
}

\author{
Wen-Chi CHIANG and Chia-Hui CHIU \\ National Chung Cheng University
}

\begin{abstract}
Extensive previous research has focused on the development of children's conceptual organization and its effects on children's veridical memory. The present research investigated whether the structure of children's category knowledge also affected their false memories using categorical lists in the DRM paradigm, a listlearning method commonly used in false memory research. In Experiment 1, the effects of graded category structure were examined with both third and sixth graders by accessing false memories for unstudied critical items of differing output dominance in category production. In Experiment 2, the effects of hierarchical category structure were examined with third graders by manipulating the hierarchical level of studied list items (basic or subordinate) and unstudied critical items (superordinate, basic, or subordinate). The results show that by age 10, the graded and hierarchical structure is present in children's conceptual organization and affects formation of false memories. Theoretical implications of the present findings are discussed.
\end{abstract}

Key words: category structure, false memory, list learning paradigm, memory development

The organization of concepts has long been an important topic in the research of human mind: it is thought to underlie several important cognitive functions that humans constantly engage in daily lives, such as classification and memory. In consequence, acquisition of an adult-like conceptual organization became a major focus in research of cognitive development.

Extensive earlier literature suggested that children's conceptual organization underwent a shift in focus from thematic/complementary relations (e.g., dog grouped with leash) to taxonomic/similarity relations (e.g., dog grouped with cat) based on their responses in sorting objects on a table into groups (e.g., Annett, 1959; Inhelder \& Piaget, 1964; Vygotsky, 1962). Some researchers hence proposed that early in development, children's knowledge was organized in terms of schematic or script-based relationships derived from perceptual-motor experiences and real-world scenes and events (e.g., Denney, 1974; Nelson, 1974), and later beyond about age 8 and through adulthood,

Wen-Chi Chiang and Chia-Hui Chiu, Department of Psychology, National Chung Cheng University, Taiwan, R.O.C. C.-H. C. is now at Graduate Institute of Early Childhood Education, National Chengchi University, Taiwan, R.O.C. Correspondences should be addressed to W.-C. C. at Department of Psychology, National Chung Cheng University, 621 Min-Hsiung, Chia-Yi, Taiwan, R.O.C. (e-mail: psywcc@ccu.edu.tw)

This research was supported by Grant NSC90-2413-H-194-022 from National Science Council of Taiwan, R.O.C. to W.-C. C. The authors thank Yuh-Shiow Lee for valuable discussions on this work; an anonymous reviewer for helpful comments on an earlier version of this article; Tian-yoong Cheng for assistance with data processing; the children for their participation; and the staff of the elementary schools for assistance with testing arrangements. 
organized primarily in terms of similarity/taxonomic relationships (Markman, 1981). However, later studies showed that young children used taxonomic relations for sorting when a different sorting context was presented (e.g., Markman, Cox, \& Machida, 1981), when a brief training was administered (Smiley \& Brown, 1979), and when a more constrained request was given (e.g., Waxman \& Namy, 1997). These findings thus suggest that from early on both taxonomic and thematic organizations are present in children's conceptual structure, but taxonomic relations are initially used in a relatively limited range of contexts but gradually replace the use of thematic relations.

Studies of development in the organization of semantic memory showed a somewhat different pattern. Unlike the thematic-to-taxonomic shift that was limited to the context of traditional sorting tasks, an associative-to-categorical shift has been constantly and robustly demonstrated in children's responses on various memory tasks ${ }^{1}$. For instance, normative research on word-associations showed that estimates of associative strength of word pairs remained stable from the third grade to college years (Bjorklund \& Jacobs, 1984), in contrast with marked age differences found in children's estimates of categorical relatedness (e.g., Posnansky, 1978). Other studies also showed that (a) preschoolers' recognition memory was predominantly mediated by complementary, not taxonomic, relations (e.g., Scott, Serchuk, \& Mundy, 1982), that (b) associative primes facilitated preschool-aged and older children's picture naming, whereas facilitative effects of categorical primes were found only in older children (McCauley, Weil, \& Sperber, 1976), and that (c) school-aged children's recall of categorizable lists of items was organized initially by associative relations between items, and later as they grow older, by the categorical relations per se (e.g., Bjorklund \& Jacobs, 1985; Frankel \& Rollins, 1985).

Different accounts have been proposed to explain this associative-to-categorical shift. One account emphasizes age differences in conscious preference for one type of organization over the other. That is, as children get older, they become more aware of category organization as a useful memory strategy and more likely to apply it during retrieval (e.g., Hasselhorn, 1990; Schneider, 1986). Another account emphasizes age differences in the ease with which different semantic relations are activated. That is, associative relations constitute a primitive form of memory organization and can be activated more readily than categorical relations for young children, even though some categorical relations may already be present in their conceptual structure (e.g., Bjorklund \& Jacobs, 1985). Whatever the explanation, association-based organization appears to have more stable effects on children's memory performance than category-based organization.

Recent years have witnessed a significant change in theoretical approaches to human memory. The traditionally dominant approach adopted a "quantity-oriented conception" in which memory was assessed in terms of the number of items that could be retrieved over some retention interval. A more recent approach has adopted, instead, an "accuracyoriented conception" in which memory is assessed in terms of its correspondence with

\footnotetext{
${ }^{1}$ Associative relations are established upon experiences of frequent co-occurrences between items; thematic or complementary relations existing among components of a schema or a script are hence all associative in nature.
} 
past events (Koriat, Goldsmith, \& Pansky, 2000). Consistent with this change in approach, there have been a large number of false memory studies using a variety of methods. It is generally held that false memory research may provide not only tests for existing theories of memory per se, but also new insights into other memory-related cognitive functions. The common use of a recently revived list-learning method, or the Deese-Roediger-McDermott (DRM) paradigm (Deese, 1959; Roediger \& McDermott, 1995), attested such a research trend.

The standard DRM procedure is to present the participants with lists of items that are semantically associated to each other (e.g., thread, eye, sewing, sharp, ...) and all converged on a critical nonpresented item (CNI; e.g., needle), and then evaluate their false memories in recall and recognition tests. This method has been used to address theoretical issues such as single-process versus dual-process theories of memory (e.g., Arndt \& Hirshman, 1998) and decision-based versus storage-based accounts for recognition biases (e.g., Lee \& Chang, 2004; Westerberg \& Marsolek, 2003), and other related issues, such as the functional independence of bilingual storage (e.g., Kawasaki-Miyaji, Inoue, \& Yama, 2003). However, only a small number of studies have applied the paradigm to children (Brainerd, Reyna, \& Forrest, 2002; Ghetti, Qin, \& Goodman, 2002), and even fewer studies have used it to examine effects of category knowledge on children's false memory formation (Seamon, Luo, Schlegel, Greene, \& Goldenberg, 2000). This is surprising because previous research on children's veridical memories of categorizable lists has been extensive (see Bjorklund, 1987, for a review). Equally surprising is that these rich findings on children's veridical memory have played almost no role in the recent theorizations of children's false memory formation.

Given the evidence that taxonomic relations are represented in young children's conceptual structure and influence their veridical memories, it is likely that children's false memories from studying categorical materials also reflect the structure of their category knowledge, and will provide insights to theories of children's memories in general. The present research addressed these possibilities by testing children with categorical lists in the DRM paradigm. Specifically, we asked whether children's false memories were influenced by the graded category structure, as indicated by degree of output dominance of items within a category (Experiment 1), and by the hierarchical category structure, as indicated by levels of specificity, or generality, of items within a category (Experiment 2).

\section{EXPERIMENT 1}

To our knowledge, Seamon et al. (2000, Experiment 1) conducted the only experiment to date that used the DRM paradigm to examine children's false memories induced by studying strictly categorical lists. In the experiment, categorical lists of pictures were presented at study, and two within-category items of differing output dominance were used as CNIs at test. The results showed that both first- and fifth-grade children were more likely to falsely recognize CNIs from studied categories than from 
unstudied categories and to falsely recognize CNIs with high output dominance than those with low output dominance. Furthermore, there was no age difference between the first and fifth graders in the false recognition rates for CNIs, and this pattern was shown not only by half of the first and fifth graders who received the immediate test but also by the other half who received the 3-day delayed test, despite that the delayed test elicited overall higher false recognition rates than the immediate test.

The Seamon et al. experiment (2000, Experiment 1), however, did not follow the standard DRM procedure. They used lists of pictures as learning materials, instead of spoken words, and used a final recognition test only, omitting the immediate recall tests following each list's presentation. Such methodological differences posed potential difficulties for comparisons between Seamon et al.'s experiment and other studies using the standard procedure, and might have been a cause for the discrepancy in the results whereas Seamon et al.'s experiment revealed no developmental differences in false memory effects, some experiments using the original DRM (associative) lists did (Brainerd et al., 2002). The present experiment thus adopted the standard DRM procedure with categorical materials to investigate (a) whether Seamon et al.'s findings on children's false recognition for pictorial CNIs of differing output dominance extend to auditory verbal stimuli and to recall tests, and (b) whether the lack of developmental differences in false memory for categorical materials found in the Seamon et al. experiment holds when the standard DRM procedure is used ${ }^{2}$.

\section{Method}

Participants. The participants were 24 third graders (mean age: 9 years 3 months; 12 boys and 12 girls) and 24 sixth graders (mean age: 11 years 10 months; 12 boys and 12 girls). Three additional children were excluded from the analyses due to the experimenter's errors $(1$ child $)$ or to the children's excessively high error rates at tests ( 2 children). The participants in this and next experiment were all recruited from schools in Chia-Yi, Taiwan.

Materials. The study lists were all categorical lists: each list consisted of labels for members from one superordinate category exclusively. The lists were constructed as follows. First, a group of sixth graders performed a category production task on 16 superordinate categories. Next, 20 of the most frequently produced items for each category were selected and read to a group of third graders on a comprehension test. Finally, eight lists of 12 items were selected for this experiment. All list items were known to children by third grade; the items that resulted in poor comprehension or multiple readings were excluded.

For six of the eight lists (animals, fruit, furniture, music instruments, sports, and vehicles), the withinlist items were ordered in the descending order of the output dominance based on a category production norm for adults in Taiwan (Jeng, Lai, \& Liu, 1973), due to lack of such norm for school-aged children. In each list, the 2nd and the 11th items were selected as CNIs. The 6 lists were equally divided into two sets (Sets A and B). A $t$ test on the list items' output frequency counts from the norm revealed no difference between sets. The remaining two lists (national festivals, kinship terms) corresponded to categories not included in the norm, and hence were used as filler lists (data not analyzed). The recognition test consisted of 40 items, 5 from each of the eight lists, including 3 studied items (the 1st, 6 th, and 12 th item) and 2 CNIs. The study lists and test items were recorded on tapes and played at study and at test, respectively. The participants'

\footnotetext{
${ }^{2}$ In the current experiment, we tested the third and six graders, rather than the first and fifth graders as did Seamon et al. (2000, Experiment 1). This change was necessary for including a reasonably large number of categorical verbal stimuli, as demanded by the DRM procedure, that were sufficiently comprehended by the younger as well as the older participants in our experiment. As a result, the age difference in the false memory effects, if found in the current experiment between the third and sixth graders, might be relatively smaller than it would be if the first and fifth graders were tested.
} 
responses were recorded for later coding.

Design and procedure. Half of the participants listened to four lists (Set A and one filler list) and the other half listened to the other four lists. All participants received the same items on the recognition test, so the responses to items from unstudied lists by half the participants served as baselines for responses to the same items by the other participants.

The participants were tested individually in a quiet classroom at their schools. The items on a list were presented at a rate of $4 \mathrm{~s}$ per item. For each list, after the last item was presented, a 90-s recall test was given. The presentation order of lists was counterbalanced across participants following a Latin square design. After the last study list was recalled, the participants were given a series of simple arithmetic problems to solve (for 1-2 min) as a distraction task before they received a self-paced final recognition test. The test items were presented in a random order with the constraint that no items from the same list were presented consecutively. The entire experimental session lasted for about $20 \mathrm{~min}$.

\section{Results}

Table 1 presents the results of free recall and final recognition. All analyses reported in this paper used an alpha level of .05 unless otherwise specified.

Recall. A 2 (grade) $\times 2$ (item type: list item, CNI) analysis of variance (ANOVA) were conducted to compare the probability of correct recall with that of false recall. The results revealed a significant effect of item type $\left(F(1,46)=996.50, M S_{\mathrm{e}}=.01\right)$ and a marginal effect of grade $\left(F(1,46)=3.89, M S_{\mathrm{e}}=.01, p=.055\right)$, which were qualified by a marginal item type $\times$ grade interaction $\left(F(1,46)=3.77, M S_{\mathrm{e}}=.01, p=.058\right)$. Post hoc comparisons revealed that the sixth graders were more likely to recall lists items than the third graders $\left(F(1,46)=10.00, M S_{\mathrm{e}}=.01\right)$, but not so for CNIs $(F<1)$.

To examine whether children's false recall of CNIs depended on the output dominance level of the items, a 2 (grade) $\times 2$ (level of output dominance) ANOVA on the false recall rates was conducted. The results revealed that high-output-dominance CNIs were more likely to be falsely recalled than low-output-dominance CNIs ( $F(1$, 46) $\left.=18.29, M S_{\mathrm{e}}=.01\right)$.

Recognition. Because the participants rarely positively recognized list items or CNIs from the unstudied lists (all rates $<.01$ ), the recognition rates for list items and CNIs were corrected by subtracting respective baseline rates and used in subsequent analyses unless specified otherwise. A 2 (grade) $\times 2$ (item type) ANOVA was conducted to compare the hit rates with the false alarm rates. Similar to the results of recall, the effect of item type was significant $\left(F(1,46)=370.25, M S_{\mathrm{e}}=.03\right)$. Unlike recall, however, neither the effect of grade nor the item type $\times$ grade interaction was significant $(F \mathrm{~s}<1)$.

A 2 (grade) $\times 2$ (level of output dominance) ANOVA was conducted on corrected false alarm rates. The pattern was similar to that of recall. Only the main effect of output dominance level was significant $\left(F(1,46)=27.51, M S_{\mathrm{e}}=.04\right)$, indicating that highoutput-dominance CNIs elicited more false recognition than low-output-dominance CNIs. Additional analyses showed greater false alarm rates for CNIs from studied categories than from unstudied categories $\left(F(1,47)=58.41, M S_{\mathrm{e}}=.02\right)$, both for high-outputdominance CNIs $\left(.34\right.$ vs. $\left..00 ; F(1,47)=67.21, M S_{\mathrm{e}}=.04\right)$ and for low-output-dominance CNIs $\left(.15\right.$ vs. $\left..01 ; F(1,47)=19.92, M S_{\mathrm{e}}=.02\right)$, demonstrating the false memory effects. 
Table 1. Mean Proportions of Positive Responses on Free Recall and Final Recognition as a Function of Grade and Type of Item in Experiment 1

\begin{tabular}{lcc}
\hline & \multicolumn{2}{c}{ Grade } \\
\cline { 2 - 3 } Type of item & Third & Sixth \\
\cline { 2 - 3 } List item & Free recall & .78 \\
CNI & .69 & .14 \\
High OD & .10 & .00 \\
Low OD & .03 & .88 \\
List item & Final recognition & \\
CNI & .85 & .33 \\
High OD & .35 & .15 \\
Low OD & .13 & \\
\hline
\end{tabular}

Note. All proportions of recognition responses were corrected by subtracting respective baseline rates (i.e., rates of positive responses to items from the unstudied set of lists). $\mathrm{CNI}=$ critical nonpresented items that were pre-designated as the study lists were constructed. $\mathrm{OD}=$ output dominance in category production responses.

\section{Discussion}

The results of false recognition in the current experiment showed a pattern similar to that found in Seamon et al.'s (2000) Experiment 1: children of both grades showed higher false alarm rates to CNIs of the studied lists than to CNIs of unstudied lists, and to high output-dominance CNIs than to low output-dominance CNIs. The results of false recall revealed a pattern consistent with that of false recognition: the likelihood of intrusion at recall was greater for CNIs of studied than of unstudied lists and greater for CNIs of high than of low output dominance. These results thus extended Seamon et al.'s findings to conditions that followed the standard DRM procedure, where categorical lists of spoken words were used as study materials and false memory assessed by immediate recall tests as well as a final recognition test.

Furthermore, the current experiment found no developmental differences in false memory: children of both third and sixth grades showed an identical pattern of false memory in recall tests and in the final recognition test. These results show that lack of developmental differences in false memory found in Seamon et al.'s (2000) Experiment 1 holds even when the standard DRM procedure is used, and suggest that false memory elicited by categorical lists may be less sensitive to age differences than that elicited by the original DRM lists. However, it must be noted that the current experiment tested the third and sixth graders, who were not far apart in age, and used lists of items that were well known to the younger age group, and thus did not rule out the possibility that age 
difference in false memory elicited by categorical lists will emerge when groups of children further apart in age are tested or when lists of items less well known to the younger children are used.

At any rate, the most important finding from the current experiment is that children's false memories after studying categorical lists are influenced by the graded category structure, as CNIs of high output dominance within categories are more likely to produce false memories than CNIs of low output dominance. In the next experiment, we addressed whether and how another property of categories, the hierarchical structure, also affected children's false memory.

\section{EXPERIMENT 2}

Extensive research has shown that categories are organized hierarchically in the conceptual structure of adults and children. Much evidence for hierarchical category structure came from phenomena of basic-level advantage: concepts at a middle level of generality (e.g., dog) were privileged relative to concepts at a more general (superordinate) level (e.g., animal) and concepts at a more specific (subordinate) level (e.g., hound) in various cognitive tasks (e.g., Jolicoeur, Gluck, \& Kosslyn, 1984; Rosch, Mervis, Gray, Johnson, \& Boyes-Braem, 1976; see Lassaline, Wisniewski, \& Medin, 1992, for a review). A prominent explanation is that relative to categories at other levels, basic-level categories are most differentiated, as within-category members are highly similar and cross-category members highly dissimilar (e.g., Murphy \& Brownell, 1985; Rosch et al., 1976). Developmental studies provided additional evidence: children tended to acquire basic-level labels earlier than superordinate or subordinate labels (e.g., Mervis $\&$ Crisafi, 1982; Rosch et al., 1976). However, other studies using nonverbal sorting tasks have shown that infants form superordinate categories earlier than basic-level categories (e.g., Mandler, Bauer, \& McDonough, 1991). Nevertheless, there is clear evidence that by nine years of age children are capable of sorting at all levels of generality (Saxby \& Anglin, 1983).

The basic-level advantage is also evident in episodic memory and, of particular relevance to the present research, in formation of false memories. In a recent study by Pansky and Koriat (2004), participants read a story in which nine categorically unrelated critical items appeared in either the superordinate, basic, or subordinate level (e.g., clothes, pants, jeans). The results of memory tests revealed a bi-directional symmetrical memory distortion, i.e., vertical shifts from different levels of studied items to basic-level responses, indicated by false recall and recognition, suggesting that the basic level is privileged for retaining episodic information in memory.

In Experiment 2, we investigated whether and how the hierarchical category structure influence children's false memory formation. The DRM paradigm was again adopted and the categorical lists were constructed as follows. For each superordinate category (e.g., animal), two lists were formed and used at study - one consisting of only basic-level items (e.g., horse, cattle, bear, ...) and the other only subordinate items (e.g., 
zebra, water buffalo, polar bear, ...) - and a basic-level item (e.g., dog), one of its subordinate (e.g., hound), and the superordinate label (e.g., animal) were designated as CNIs to assess false memories ${ }^{3}$. The use of DRM paradigm with categorical lists specifically designed as such had at least two advantages. First, it allowed us to compare the current experiment more directly with recent false memory research in which the DRM paradigm had been widely used, and with previous studies on children's memory (true and false) for categorical materials which also largely used list-learning methods. Second, it enabled us to investigate memory distortion not only in the form of vertical shifts (Pansky \& Koriat, 2004), but in the form of horizontal shifts as well. Whereas vertical shifts refer to false memory for unstudied items from levels that are vertically related to (i.e., more general or more specific than) the level of studied list items, horizontal shifts refer to false memory for unstudied items from the same level as the level of studied list items. By the present experimental design, if children's false memories were influenced by the hierarchical category structure, then after studying basic- and subordinate-level lists, false memories should occur as (a) upward vertical shifts (false memories either for superordinate- or for basic-level CNIs), and as (b) horizontal shifts, indicating that vertical links from the studied level to upper levels and horizontal links within the studied level had both been activated ${ }^{4}$.

Furthermore, the present design can also provide implications for existing theories for false memory formation. For example, according to fuzzy-trace theory (e.g., Brainerd \& Reyna, 1998), one of the leading theories for false memories created in the DRM paradigm, dissociated representations are formed for studied stimuli: the verbatim traces represent the exact surface form of the stimuli and the gist traces represent the meaning (or theme) of them. Because the verbatim traces of studied list items are lost more rapidly and more difficult to retrieve than their gist traces over retentions, false memories for the CNI of an associative DRM list or a categorical list are likely to occur when the unstudied item is consistent with the gist traces of the studied items in the list. However, fuzzy-trace theory is unspecified with respect to whether the gist representation of a categorical list corresponds best to an item at the superordinate level or, alternatively, to a frequently

\footnotetext{
${ }^{3}$ It should be noted that in the present research, basic and subordinate level items were defined in a general and relative sense, not in an absolute or experimentally proven sense. Basic-level items under a superordinate category were selected mainly from materials used by Rosch et al. (1976) and from the category production norm (Jeng et al., 1973). In general, these items were commonly used labels that referred to typical categories at an intermediate level of generality, relative to the superordinate category. Once these intermediate categories were determined, we either selected or generated subordinate level items that were labels for categories at a relatively more specific level. For all subordinate items, the sentence "A [subordinate item] is a kind of [basic level item]" must be judged to be true before they were accepted for consideration as list items.

${ }^{4}$ Pansky and Koriat (2004) did not include in their results memory distortion in the form of horizontal shifts because the proportion was omissible. The small proportion of horizontal shifts was likely due to the use of single items from different superordinate categories. In contrast, the current experiment, as many others using the DRM paradigm, used multiple items (in lists) from different superordinate categories, and thus might reveal an elevated level of false memories in the form of horizontal shifts. Similarly, the present experiment might reveal false memories in the form of vertical shifts to superordinate level, even though Pansky and Koriat (2004) found no evidence for vertical shifts in such a form, but only vertical shifts converging to the basic level.
} 
cited basic-level item, or a prototype, of the superordinate category. This issue could be addressed in the present experiment by examining the likelihood of false memory occurrence for unstudied but list-related superordinate items versus basic-level items irrespective of the studied items being at basic or subordinate level.

\section{Method}

Participants. The participants were 32 third graders (mean age: 9 years 8 months; 16 boys and 16 girls). No children had participated in Experiment 1. Five additional children were excluded from analyses due to excessively high error rates at tests ( 3 children) or to failure in the recognition pretest ( 2 children; details described below).

Materials and design. The study lists were categorical lists, constructed as follows. First, we selected 28 superordiante categories and, for each, listed the superordinate category label and labels for its members at the basic and subordinate levels. Next, we checked these labels against a database of words commonly accessible to elementary school children (Ministry of Education, R.O.C., 2000) to filter out words that were unlikely known to children. The remaining items were then tested on a group of 21 third graders to further filter out those resulting in multiple readings or poor comprehension. Eight key categories were selected eventually (i.e., animal, clothing, furniture, kitchenware, music instrument, snack, stationery, and vehicle).

For each of the key categories, two types of lists were formed, one consisting of 11 basic level items (the $b$ list), the other 11 subordinate items (the $s$ list). The items on each list were ordered in the descending order of frequency counts according to the word database (Ministry of Education, R.O.C., 2000). The 2nd to 11 th items in each list were used as study items, and the 1st item as the CNI. The eight categories were equally divided into two sets (Sets $\mathrm{C}$ and D). The $t$ tests on the frequency counts of list items showed no differences between sets on either the $b$ lists or the $s$ lists. Each participant received 4 lists from either Set $\mathrm{C}$ or D, including $2 b$ lists and $2 s$ lists, in the order of $b, s, s, b$, or of $s, b, b, s$. The presentation order of the four categories in each set was counterbalanced across participants following a Latin square design.

Two additional lists were used as filler lists (data not analyzed), with each list consisting of items that were labels for categories at differing hierarchical levels ( 4 superordinates, 3 basic-levels, and 3 subordinates) and that all were categorically unrelated to each other and to any item from the key categories. In addition, one filler list was always the first presented list at study and the other always the last. These arrangements served to reduce the potential primacy and recency effects on the participants' memories for the key lists, and to mask an impression that superordinate labels never appeared in the key lists.

The recognition test consisted of 52 items, including 3 studied items from each of the four studied lists (the 2nd, 7th, and 11th) and 2 CNIs from each of the four studied categories (its superordinate label and the 1 st item of either its $b$ list or $s$ list), 5 corresponding items from each of the four unstudied lists, 4 studied items from each filler list, and 4 novel items unrelated to any list item from Sets C and D. In response to the manipulation of hierarchical level of list items at study, the recognition test was manipulated as follows: regardless of the actual hierarchical level of the four key lists each participant studied (in the order of either $b$, $s, s, b$, or $s, b, b, s)$, the CNIs were always the 1 st item of the $s, b, s$, and $b$ lists of the first to fourth studied categories respectively, and their superordinate labels. This allowed us to examine false recognition in all four possible study-test pairings within and cross the basic and subordinate levels. In addition, the positions of the superordinate CNIs on the recognition test were also manipulated, with them appearing either before or after other items from the corresponding categories.

Apparatus and procedure. The apparatus and procedure were identical to those in Experiment 1 except that the recall test was shortened to a 60 -s period. This modification enabled the testing to proceed at a smoother pace and the entire experimental session to last for about $20 \mathrm{~min}$ as in Experiment 1.

Because the final recognition test included superordinate CNIs, it was crucial to emphasize to children that a test item must be a word actually heard at study to be accepted as an "old" item, and that any unheard higher-level labels for the studied items should be rejected. For this purpose, the participants received a pretest, prior to the final recognition test, on a brief list of country names they heard in a practice before the experiment began. The pretest consisted of a studied country name, a unstudied country name, and the superordinate label "country" for the practice list. In cases where false recognition occurred, corrective feedbacks were given, and the practice list was re-presented and the pretest repeated. Data from the participants who failed the repeated pretest were excluded from the analyses. 
Table 2. Mean Proportions of Positive Responses on Free Recall and Final Recognition as a Function of Level of Studied List Items and Type of Item in Experiment 2

\begin{tabular}{lcc}
\hline \multirow{2}{*}{ Type of item } & \multicolumn{2}{c}{ Level of studied list items } \\
\cline { 2 - 3 } & Fasic level & Subordinate level \\
\hline & .63 & .58 \\
List item & .03 & .02 \\
CNI (basic level) & & \\
RNI* & 0.44 & 0.38 \\
Basic level & 0.06 & 0.47 \\
Subordinate level &
\end{tabular}

Final recognition

List item

CNI

Basic level

Subordinate level

Superordinate level

First at test

Note. All proportions of recognition responses were corrected by subtracting respective baseline rates (i.e., rates of positive responses to items from the unstudied set of lists). $\mathrm{CNI}=$ critical nonpresented items that were pre-designated as the study lists were constructed. RNI = related nonpresented items that were not studied but categorically related to the study lists, including the pre-designated CNIs. * Recall of RNI was measured in terms of frequency of ocurrences per list.

\section{Results}

Recall. Recall results are presented in the upper half of Table 2. Children were more likely to recall studied items from the $b$ lists than the $s$ lists, although the effect was only marginal $\left(F(1,31)=3.92, M S_{\mathrm{e}}=.01, p=.057\right)$. False recall of CNIs occurred only at the basic level and was equally rare for the $b$ lists and $s$ lists $(F=1)$, and never occurred the other two levels.

As one purpose of the current experiment was to examine the gist-based accounts for false memory formation, an additional measure of the gist-based effects on false recall was adopted in addition to the recall rates of pre-designated CNIs. For each studied category, mean frequencies of categorically related items that were recalled but not included in the studied list (including the pre-designated CNIs), or related nonpresented items (RNIs), were calculated separately at the basic and subordinate levels. The RNIs' levels were determined by the same criteria used to construct the study lists at differing levels (see footnote 2). This measure of false recall, in which all possible gist-consistent 
items were taken into account, is likely to be more sensitive to the gist-based effects than recall rates of only the CNIs. A 2 (level of studied list: basic, subordinate) $\times 2$ (level of recalled RNI: basic, subordinate) ANOVA on mean frequency of RNIs occurring in recall yielded a significant main effect of study level $\left(F(1,31)=6.81, M S_{\mathrm{e}}=.14\right)$, which was qualified by a significant study level $\times$ recall level interaction $\left(F(1,31)=5.74, M S_{\mathrm{e}}=.31\right)$. Post hoc Tukey HSD tests revealed that related items were falsely recalled more frequently in the study-recall level-congruent conditions $\left(M_{\text {basic-basic }}=0.44 ; M_{\text {subordinate- }}\right.$ subordinate $=0.47)$ than in one of the incongruent conditions $\left(M_{\text {basic-subordinate }}=0.06\right), p \mathrm{~s} \leq .05$. No other comparisons were significant $(p \mathrm{~s}>.13)$.

Recognition. Recognition results are presented in the lower half of Table 2. Children showed identical recognition performance on studied items regardless of the level of studied lists (basic vs. subordinate) or the position of superordinate CNIs at test (first vs. last), as indicated by a $2 \times 2$ ANOVA on baseline-corrected hit rates, $F \mathrm{~s}<1$.

A 2 (level of studied lists) $\times 2$ (position of superordinate CNIs at test) $\times 3$ (level of CNIs at test: superordinate, basic, or subordinate) ANOVA was conducted on corrected false alarm rates for CNIs. The results revealed a significant effect of level of CNI ( $F(1$, $\left.60)=3.70, M S_{\mathrm{e}}=.09\right)$. Post hoc comparisons showed that false alarm rates were significantly higher for basic-level CNIs $(M=.16)$ than for subordinate CNIs $(M=.02)$, while neither differed from that for superordinate CNIs $(M=.08)$. However, the 3-way interaction was also significant $\left(F(1,60)=3.55, M S_{\mathrm{e}}=.08\right)$, mainly due to a differing response pattern for superordinate CNIs. Similar ANOVAs were then conducted separately for CNIs at different levels to clarify this interaction.

A $2 \times 2$ ANOVA conducted on corrected false alarm rates for superordinate CNIs revealed a significant interaction between level of studied lists and position at test $(F(1$, $\left.30)=4.97, M S_{\mathrm{e}}=.08\right)$. Neither main effect was significant $\left(F_{\mathrm{S}}<1\right)$. False recognition of superordinate CNIs for $b$ lists was more likely to occur when the CNIs appeared first than last at test; the reverse was true for $s$ lists. However, post hoc analyses showed that these trends were unreliable $(p s>.08)$.

A $2 \times 2 \times 2$ ANOVA conducted on corrected false alarm rates for CNIs at basic versus subordinate level showed that basic-level CNIs were more likely to be falsely recognized than subordinate CNIs $\left(F(1,30)=7.28, M S_{\mathrm{e}}=.09\right)$. Planned comparisons, however, showed that this effect of level of CNIs occurred for $b$ lists only, not for $s$ lists. No other main effects or interactions were significant ( $p \mathrm{~s}>.07)$.

\section{Discussion}

Comparing with Experiment 1, false memories for CNIs were reduced considerably in Experiment 2: children rarely recalled the basic-level CNIs and never the superordinate or subordinate CNIs; false recognition of CNIs was also reduced. One possible explanation for the reduction in false recall has to do with a methodological difference between Experiments 1 and 2. In Experiment 2, each participant studied both $b$ and $s$ lists, with the $s$ lists consisting of items more differentiated and distinctive from each other than the $b$ lists and the lists used in Experiment 1. Such enhanced item-specific information in some lists could have facilitated distinctive processing and prevented relational 
processing (i.e., processing of similarities among items that would increase false memory) for all lists during encoding (R.E. Smith \& Hunt, 1998). This account also explains in part the reduction in false recognition in Experiment 2. The reduced false recognition also can be explained in part by warning at test (indirectly given in the current experiment via the recognition pretest on the practice list and corrective feedback) and in part by the use of distinctiveness heuristic - a response mode based on a metamemory awareness that recognition of studied items must include recollection of distinctive details (Schacter, Israel, \& Racine, 1999); CNIs thus become more likely to be rejected because distinctive details were unlikely to be retrieved for them.

Analyses of another false-recall measure, frequency of RNIs, revealed more important results. Studying $b$ lists induced more false recall of basic-level RNIs (horizontal shifts at basic level) than subordinate RNIs (vertical shifts away from basic level). Furthermore, studying $s$ lists induced false recall of basic-level RNIs (vertical shifts toward basic level) as well as subordinate RNIs (horizontal shifts at subordinate level), both at a comparable level of occurrence to basic-level horizontal shifts. These patterns of results suggest that children's false memories are influenced by the hierarchical category structure and provide some implications for fuzzy-trace theory.

Consistent with fuzzy-trace theory, both vertical shifts toward, and horizontal shifts at, the basic level support the notion that the gist of a categorical list corresponds to a representative basic-level item of the superordiante category from which the list items are derived. Moreover, although the current results cannot rule out that a categorical list's gist also corresponds to an item representing the list at the superordinate level, the results that superordinate CNIs are never falsely recalled and are no more likely to be falsely recognized than basic-level CNIs suggest that the gist of a studied list is not best characterized as corresponding to its superordinate label. An additional and important finding is that horizontal shifts are sensitive to the encoded level. Unlike false memories toward or at the basic level, horizontal shifts at the subordinate level found here are not readily explained by fuzzy-trace theory. This pattern indicates that, with regard to fuzzytrace theory, the gist representation of a categorical list formed at encoding must also include the specific hierarchical-level information common to all the list items.

Explanations presented above are consistent with the view that structure of category knowledge plays some role in creating false memories for categorical lists. An alternative account, derived mainly from findings on the standard DRM lists, is the activationmonitoring theory - false memories occur because CNIs are automatically activated through associative processes at encoding and become undistinguished from activation of actually studied items when monitoring processes are compromised (e.g., Roediger, Watson, McDermott, \& Gallo, 2001). Consistent with this theory, Roediger et al. (2001) found that variance in false recall of CNIs were accounted for largely by the associative strength from list items to the CNI (positive correlation) and by probability of veridical recall of list items (negative correlation). It is thus possible that false memories for categorical CNIs result from similar associative processes, and that category structure plays no important role.

However, there is evidence against this view. A recent study showed that associative 
(DRM) lists and categorical lists could elicit comparable levels of false recall, but through different processes (S.M. Smith, Gerkens, Pierce, \& Choi, 2002). First, categorical CNIs were given as associative responses to categorical list items far less frequently than associative CNIs to associative list items. Furthermore, indirect priming for CNIs were found after associative lists, but not categorical lists, were studied, suggesting that for associative lists, false memories of CNIs occurred because of automatic activation of CNIs at encoding, whereas for categorical lists, false memories occurred because of the use of semantic knowledge at test.

Similarly, associative processes at encoding cannot account for some of the current results. It is highly unlikely that subordinate RNIs were activated when the subordinate lists were studied as such specific items rarely occurred in associative responses, and yet subordinate RNIs were falsely recalled just as frequently as less specific, basic-level RNIs, which were more likely to occur in associative responses. This, however, does not mean that associative processes play no role in creating false memories for categorical lists, but suggests that they are insufficient.

The current findings can be better explained by gist-based accounts. Previous research on children's veridical memories for categorizable lists of items have shown that clustering in recall increases as the use of categorical relations at retrieval becomes as easy as the use of associative relations (e.g., Bjorklund \& Jacobs, 1985) or becomes a preferred strategy for retrieval (e.g., Hasselhorn, 1990). In parallel, children's false memories for gist-consistent but unstudied categorical items may arise as children become better at abstracting the gist of categorical lists (e.g., Brainerd et al., 2002) or better at utilizing the categorical gist for retrieval.

In conclusion, using the DRM paradigm and categorical lists, the present research shows that by age 10, children's concepts are organized in a graded and hierarchical structure and that this conceptual organization plays a role in false memory creation.

\section{REFERENCES}

Annett, M. 1959. The classification of instances of four common class concepts by children and adults. British Journal of Psychology, 29, 233-236.

Arndt, J., \& Hirshman, E. 1998. True and false recognition in MINERVA2: Explanations from a global matching perspective. Journal of Memory and Language, 39, 371-391.

Bjorklund, D. F. 1987. How age changes in knowledge base contribute to the development of children's memory. Developmental Review, 7, 93-130.

Bjorklund, D. F., \& Jacobs, J. W. 1984. A developmental examination of ratings of associative strength. Behavior Research Methods, Instruments \& Computers, 16, 568-569.

Bjorklund, D. F., \& Jacobs, J. W. 1985. Associative and categorical processes in children's memory: The role of automaticity in the development of organization in free recall. Journal of Experimental Child Psychology, 39, 599-617.

Brainerd, C. J., \& Reyna, V. F. 1998. Fuzzy-trace theory and children's false memories. Journal of Experimental Child Psychology, 71, 81-129.

Brainerd, C. J., Reyna, V. F., \& Forrest, T. J. 2002. Are young children susceptible to the false-memory illusion? Child Development, 73, 1363-1377.

Deese, J. 1959. On the prediction of occurrence of particular verbal intrusions in immediate recall. Journal 
of Experimental Psychology, 58, 17-22.

Denney, N. 1974. Evidence for developmental changes in categorization criteria for children and adults. Human Development, 17, 41-53.

Frankel, M. T., \& Rollins, H. A. 1985. Associative and categorical hypotheses of organization in the free recall of adults and children. Journal of Experimental Child Psychology, 40, 304-318.

Ghetti, S., Qin, J., \& Goodman, G. S. 2002. False memories in children and adults: Age, distinctiveness, and subjective experience. Developmental Psychology, 38, 705-718.

Hasselhorn, M. 1990. The emergence of strategic knowledge activation in categorical clustering during retrieval. Journal of Experimental Child Psychology, 50, 59-80.

Inhelder, B., \& Piaget, J. 1964. The early growth of logic in the child. New York: Norton.

Jeng, C.-I., Lai, M.-W., \& Liu, I.-M. 1973. Category norms in Chinese and English from bilingual subjects. Acta Psychologica Taiwanica, 15, 81-153.

Jolicoeur, P., Gluck, M. A., \& Kosslyn, S. M. 1984. Pictures and names: Making the connection. Cognitive Psychology, 16, 243-275.

Kawasaki-Miyaji, Y., Inoue, T., \& Yama, H. 2003. Cross-linguistic false recognition: How do Japanesedominant bilinguals process two languages: Japanese and English? Psychologia, 46, 255-267.

Koriat, A., Goldsmith, M., \& Pansky, A. 2000. Toward a psychology of memory accuracy. Annual Review of Psychology, 51, 481-537.

Lassaline, M. E., Wisniewski, E. J., \& Medin, D. L. 1992. Basic levels in artificial and natural categories: Are all basic levels created equal? In B. Burns (Ed.), Percepts, concepts, and categories (pp. 327 378). Amsterdam: Elsevier Science.

Lee, Y.-S., \& Chang, S.-C. 2004. Effects of criterion shift on false memory. Psychologia, 47, 191-202.

Mandler, J. M., Bauer, P. J., \& McDonough, L. 1991. Separating the sheets from the goats: Differentiatating global categories. Cognitive Psychology, 23, 263-298.

Markman, E. M. 1981. Two different principles of conceptual organization. In M.E. Lamb \& A.L. Brown (Eds.), Advances in developmental psychology (pp. 199-236). Hillsdale, NJ: Erlbaum.

Markman, E. M., Cox, B., \& Machida, S. 1981. The standard object-sorting task as a measure of conceptual organization. Developmental Psychology, 17, 115-117.

McCauley, C., Weil, C. M., \& Sperber, R. D. 1976. The development of memory structure as reflected by semantic-priming effects. Journal of Experimental Child Psychology, 22, 511-518.

Mervis, C. B., \& Crisafi, M. A. 1982. Order of acquisition of subordinate-, basic-, and superordinate-level categories. Child Development, 53, 258-266.

Ministry of Education, R. O. C. 2000. Guoxiao xuetong changyong zici diaocha baogaoshu [A survey of frequently used characters and words by elementary school children]. Taipei: Mandarin Promotion Council, Ministry of Education, R.O.C. (In Chinese.)

Murphy, G. L., \& Brownell, H. H. 1985. Category differentiation in object recognition: Typicality constraints on the basic category advantage. Journal of Experimental Psychology: Learning, Memory, and Cognition, 11, 70-84.

Nelson, K. 1974. Concept, word, and sentence: Inter-relations in acquisition and development. Psychological Review, 81, 267-285.

Pansky, A., \& Koriat, A. 2004. The basic-level convergence effect in memory distortions. Psychological Science, 15, 52-59.

Posnansky, C. J. 1978. Category norms for verbal items in 25 categories for children in grades 2-6. Behavior Research Methods \& Instrumentation, 10, 819-832.

Roediger, H. L. III, \& McDermott, K. B. 1995. Creating false memories: Remembering words not presented in lists. Journal of Experimental Psychology: Learning, Memory, and Cognition, 21, 803-814.

Roediger, H. L., Watson, J. M., McDermott, K. B., \& Gallo, D. A. 2001. Factors that determine false recall: A multiple regression analysis. Psychonomic Bulletin \& Review, 8, 385-407.

Rosch, E., Mervis, C. B., Gray, W. D., Johnson, D. M., \& Boyes-Braem, P. 1976. Basic objects in natural categories. Cognitive Psychology, 8, 382-439.

Saxby, L., \& Anglin, J. M. 1983. Children's sorting of objects from categories of differing levels of generality. Journal of Genetic Psychology, 143, 123-137.

Schacter, D. L., Israel, L., \& Racine, C. 1999. Suppressing false recognition in younger and older adults: The distinctiveness heuristic. Journal of Memory and Language, 40, 1-24. 
Schneider, W. 1986. The role of conceptual knowledge and metamemory in the development of organizational processes in memory. Journal of Experimental Child Psychology, 42, 318-336.

Scott, M. S., Serchuk, R., \& Mundy, P. 1982. Taxonomic and complementary picture pairs: Ability in two- to five-year olds. International Journal of Behavioral Development, 5, 243-256.

Seamon, J. G., Luo, C. R., Schlegel, S. E., Greene, S. E., \& Goldenberg, A. B. 2000. False memory for categorized pictures and words: The category associates procedure for studying memory errors in children and adults. Journal of Memory and Language, 42, 120-146.

Smiley, S. S., \& Brown, A. L. 1979. Conceptual preference for thematic or taxonomic relations: A nonmonotonic age trend from preschool to old age. Journal of Experimental Child Psychology, 28, 249-257.

Smith, S. M., Gerkens, D. R., Pierce, B. H., \& Choi, H. 2002. The roles of associative responses at study and semantically guided recollection at test in false memory: The Kirkpatrick and Deese hypotheses. Journal of Memory and Languages, 47, 436-447.

Smith, R. E., \& Hunt, R. R. 1998. Presentation modality affects false memory. Psychonomic Bulletin \& Review, 5, 710-715.

Vygotsky, L. S. 1962. Thought and language. Cambridge, MA: MIT Press.

Waxman, S. R., \& Namy, L. L. 1997. Challenging the notion of a thematic preference in young children. Developmental Psychology, 33, 555-567.

Westerberg, C. E., \& Marsolek, C. J. 2003. Sensitivity reductions in false recognition: A measure of false memories with stronger theoretical implications. Journal of Experimental Psychology: Learning, Memory, and Cognition, 29, 747-759.

(Manuscript received January 5, 2005; Revision accepted March 14, 2006) 